\title{
Nonequilibrium fluctuations during diffusion in liquid layers
}

\author{
Doriano Brogioli ${ }^{1}$ and Alberto Vailati ${ }^{2, *}$ \\ ${ }^{1}$ Universität Bremen, Energiespeicher- und Energiewandlersysteme, Bibliothekstraße 1, 28359 Bremen, Germany \\ ${ }^{2}$ Dipartimento di Fisica, Università degli Studi di Milano, I-20133 Milano, Italy
}

(Received 19 January 2017; published 18 July 2017)

\begin{abstract}
Theoretical analysis and experiments have provided compelling evidence of the presence of long-range nonequilibrium concentration fluctuations during diffusion processes in fluids. In this paper, we investigate the dependence of the features of the fluctuations from the dimensionality of the system. In three-dimensional fluids the amplitude of nonequilibrium fluctuations can become several orders of magnitude larger than that of equilibrium fluctuations. Notwithstanding that, the amplitude of nonequilibrium fluctuations remains small with respect to the concentration difference driving the diffusion process. By extending the theory to two-dimensional systems, such as liquid monolayers and bilayers, we show that the amplitude of the fluctuations becomes much stronger than in three-dimensional systems. We investigate the properties of the fronts of diffusion and show that they have a self-affine structure characterized by a Hurst exponent $H=1$. We discuss the implications of these results for diffusion in liquid crystals and in cellular membranes of living organisms.
\end{abstract}

DOI: 10.1103/PhysRevE.96.012136

\section{INTRODUCTION}

A multicomponent fluid at equilibrium undergoes spontaneous local concentration fluctuations representing small perturbations of the macroscopic state. The presence of a concentration gradient puts the fluid in a nonequilibrium condition, characterized by the presence of a macroscopic diffusive mass flux [1]. Nonequilibrium fluctuations orders of magnitude larger than the equilibrium ones have been predicted theoretically [2-7] and reported experimentally [8-11] during diffusion between couples of ordinary miscible fluids. These nonequilibrium fluctuations are originated by thermally excited velocity fluctuations, which give rise to vortices at mesoscopic length scales displacing volumes of fluid in layers with different concentration. Theory and experiments have shown that in the absence of gravity the fluctuations do not exhibit any characteristic length scale, beyond the molecular and macroscopic ones that provide intrinsic lower and upper bounds for the size of fluctuations [12,13]. On Earth, a characteristic length scale is determined by the presence of the gravity force, which quenches long-wavelength fluctuations [5,6,8,9,14-16].

Linearized hydrodynamics [17] represents an effective quantitative model to describe the structure and the dynamics of the fluctuations [7]. As in any linearized model, the key assumption is that fluctuations represent small perturbations of the macroscopic variables. This assumption is confirmed by experiments and simulations, even under microgravity conditions where the amplitude of the fluctuations is not damped by external forces $[12,13,18-20]$. Notwithstanding the effectiveness of linearized hydrodynamics, recent theoretical work has found that the cumulative contribution of secondorder terms to the equations is far from being negligible. On the contrary, the cumulative contribution of the microscopic mass currents determined by nonequilibrium fluctuations accounts for the whole Fick's flux [21-23]. Therefore, notwithstanding the fact that nonequilibrium fluctuations are small perturba-

\footnotetext{
*Corresponding author: alberto.vailati@unimi.it
}

tions of a macroscopic condition, they represent the essence of the diffusion process at the mesoscopic scale.

Second-order terms also play an important role in determining emergent properties of nonequilibrium fluids that cannot be described by linearized hydrodynamics. The most striking example along this line is the recent theoretical prediction of Casimir-like forces determined by nonequilibrium concentration fluctuations under confinement [24-26]. This effect is similar to the critical Casimir effect, predicted by Fisher and De Gennes [27] for a near-critical binary mixture under confinement and recently confirmed experimentally $[28,29]$. In both cases, the presence of long-ranged fluctuations gives rise to an imbalance of pressure under confinement. In the case of the critical Casimir effect, fluctuations become long ranged only close to a critical point, whereas for a nonequilibrium liquid the long-range fluctuations occur under generic conditions [30], without the need to fine-tune the parameters of the system.

Until now, the theoretical and experimental investigation of nonequilibrium fluctuations has been mostly limited to three dimensional fluids. Traditionally, the theoretical description of a thin layer of liquid is achieved by integrating the full three-dimensional (3D) hydrodynamic equations across the thickness of the layer, so to obtain a quasi-2D model. A seminal example is represented by the lubrication theory developed by Osborne Reynolds [31]. More recently, the dewetting dynamics of a thin film has been modelled effectively by integrating the linearized hydrodynamics equations across the film thickness to obtain a stochastic thin-film equation [32]. This allowed to describe the time evolution of the free surface profile of the film in 2D and to achieve a better understanding of the role of thermal fluctuations in the rupturing of the film. Lubrication theory, and other thin-film models based on the same approach, rely on the integration of the 3D hydrodynamic equations across the film thickness to achieve a description of the film at spatial wavelengths $\Lambda$ much larger than the thickness $h$ of the film. As any other hydrodynamic approach, the thin-film limit is based on the assumption that the liquid is a continuum along all the spatial directions: a small volume of fluid must always contain a large number of molecules, even 
across the film thickness. Conversely, in this work we deal with model liquid systems made by monolayers and bilayers of molecules. The thickness of the layer is constant and strictly determined by the size of the molecules. The molecules are free to diffuse in the direction parallel to the surface of the layer, but their motion in the direction perpendicular to the layer is forbidden. The continuum assumption does not hold in 3D for such liquids, since along the axis perpendicular to the liquid layer the discrete nature of the medium becomes apparent. This makes it impossible to describe this kind of system by using the thin-film approach. However, in the case of liquid layers the system can be modelled effectively as a continuum in $2 \mathrm{D}$ by revisiting the continuum assumption: A small portion of the surface of the liquid must always contain a large number of molecules. This allows us to deal with the liquid layer as a continuum in the direction parallel to its surface and as a discrete medium in the direction perpendicular to it. By contrast with liquid films, this kind of system is intrinsically two dimensional. This feature allows the direct development of the two-dimensional hydrodynamic model described in this work, without the need of averaging out one of the spatial coordinates to attain an effective two-dimensional system.

Quite interestingly, the number of molecules at the surface of a liquid layer is fixed. As a consequence, its surface tension is zero $[33,34]$ and thermocapillary and solutocapillary flows are not present. Indeed, this lack of effects determined by surface tension is a general feature of truly 2D liquids, where the absence of a bulk phase inhibits capillary effects. Examples include monolayers made of liquid crystals [35] and lipid bilayers [36]. Indeed, 2D hydrodynamics is becoming an increasingly important tool to describe the spatial-temporal dynamics of active matter in 2D to describe phenomena such as the swarming of epithelial cell monolayers [37], the development of turbulence in concentrated living sperm [38,39], and the collective dynamics of swimming bacteria [40].

Although the $3 \mathrm{D}$ hydrodynamics is not relevant for the description of the fluid layer, we consider the 3D hydrodynamics of the fluid surrounding the layer, which is often relevant in practical cases. In order to investigate the phenomenon of giant nonequilibrium fluctuations in a different dimensionality, in this work we extend linearized hydrodynamics to two-dimensional fluids. We find that in 2D, nonequilibrium fluctuations are much more intense than in $3 \mathrm{D}$ and the diffusion process is dominated by long-wavelength fluctuations. The contribution of long-wavelength fluctuations to the diffusion flux diverges for two-dimensional fluids, thus emphasizing the role of long-wavelength fluctuations in the diffusive transport in two dimensions. This is in contrast with the three-dimensional case, where the contribution to diffusion of the long wavelength fluctuations is negligible and the nonequilibrium fluctuations can be considered as the low-frequency tail of the diffusion process, which mainly occurs at the molecular level. The divergence in 2D fluids is intimately related to the well-known "Stokes paradox," which states that no bounded solutions can be found for the Stokes equation in 2D for the flow past a finite body [41]; here the paradox is described in terms of fluctuating hydrodynamics.

The diffusion process is driven by the presence of a macroscopic concentration profile inside the sample. The sets of points with equal concentration constitute the so-called isoconcentration surfaces or fronts of diffusion. Under the action of a uniform concentration gradient, and in the absence of fluctuations, these surfaces are planes perpendicular to the gradient. Nonequilibrium fluctuations give rise to a corrugation of these planar surfaces and it has been argued that in three dimensions the surfaces have a fractal structure [42,43]. Experiments performed under microgravity conditions confirmed that the mean-squared amplitude of the fluctuations as a function of wave vector exhibits a power-law behavior, a feature compatible with the presence of scale invariance, but were not able to provide a quantitative characterization of the fractal dimension $[12,13,18]$. Further theoretical work showed that the fronts of diffusion exhibit a self-affine structure rather than a self-similar one, as it is often the case of interfaces in anisotropic systems [44]. The scaling law is compatible with a fractal structure only at length scales that are not actually covered by the fluctuations (smaller than the molecular size) [19]. In the case of diffusion in 2D, we find that the scaling laws of the isoconcentration curves are not compatible with a fractal structure at any length scale. Instead, isoconcentration curves have the self-affine structure of a fractional Brownian process characterized by a Hurst exponent $H=1$ and Hausdorff dimension $d_{f}=1$ across all the length scales of the system.

The paper is organized as follows. In Sec. II we briefly review the experimental model systems where the effect of nonequilibrium fluctuations can become appreciable. In Sec. III we extend the formalism of fluctuating hydrodynamics to two-dimensional fluids by also considering the case of a liquid layer surrounded by a viscous fluid. In Sec. IV we evaluate the contribution of nonequilibrium fluctuations to the Fick's flux in two-dimensional systems. In Sec. V we evaluate the mean-square amplitude of the fluctuations. In Secs. IV and V we discuss the phenomenon of nonequilibrium fluctuations in liquid layers by taking into account the viscous drag of the fluids surrounding the film. In Sec. VI A we graphically compare the fluctuations in three- and twodimensional fluids and in liquid layers with viscous drag exerted by the surrounding gases. In Sec. VI B we evaluate the fractal dimension of the fronts of diffusion in two-dimensional fluids. In the conclusions, Sec. VII, we briefly suggest the implications of our results in understanding diffusion in cellular membranes. In the appendix we evaluate the shear force exerted by a gas under medium vacuum on a thin liquid layer.

\section{EXPERIMENTAL MODEL SYSTEMS.}

Various hydrodynamic phenomena have been investigated in two-dimensional liquids, including turbulent and laminar fluxes. Among these, freely suspended liquid layers represent a model system for the investigation of hydrodynamics in two dimensions to tackle phenomena such as diffusion $[45,46]$, turbulence [47-50], and convection [51]. In this work we are interested in the investigation of 2D model systems such as liquid monolayers and bilayers. These model systems cannot be described by using the thin-film approach, which relies on the integration of the $3 \mathrm{D}$ hydrodynamic equations across 
TABLE I. Typical values of the physical parameters for experimentally relevant systems.

\begin{tabular}{lll}
\hline \hline Property & \multicolumn{1}{c}{ Liquid crystal } & \multicolumn{1}{c}{ Lipid bilayer } \\
\hline Shear viscosity of layer $\eta_{0}$ & $5 \times 10^{-2} \mathrm{~Pa} \mathrm{~s}[35]$ & $0.3 \mathrm{~Pa} \mathrm{~s}[60]$ \\
Shear viscosity of surrounding fluid $\eta_{1}$ & $1.9 \times 10^{-5} \mathrm{~Pa} \mathrm{~s}$ (air) & $1 \times 10^{-3}$ Pa s (water) \\
Typical layer thickness $h$ & $2 \mathrm{~nm}$ & $3 \mathrm{~nm}$ \\
Typical molecular size $a$ & $0.4 \mathrm{~nm}$ & $0.4 \mathrm{~nm}$ \\
Characteristic wave vector $q_{C}$ & $3.8 \times 10^{5} \mathrm{~m}^{-1}$ & $2 \times 10^{6} \mathrm{~m}^{-1}$ \\
Characteristic wavelength $\lambda_{0}$ & $1.6 \times 10^{-5} \mathrm{~m}$ & $3 \times 10^{-6} \mathrm{~m}$ \\
\hline \hline
\end{tabular}

the thickness of the film. Instead, their theoretical description requires the development of a full $2 \mathrm{D}$ hydrodynamic model.

We consider the 3D hydrodynamics of the fluid surrounding the layer, which is often relevant in practical cases. A pure $2 \mathrm{D}$ behavior would require that the drag by the surrounding fluid is negligible; this can be actually achieved in the case of mono- or bilayers of liquid crystals suspended under vacuum (see Appendix). However, at variance with lipid bilayers, it must be noticed that liquid crystals tend to form a bulk phase, and thus present surface capillary effects, which are outside the scope of the present work; we suggest that a pure 2D behavior could be observed, e.g., by Fluorescent Recovery After Photobleaching experiments [52], in which the surface tension is almost perfectly uniform. Experiments on two-dimensional model systems have been mostly performed with liquid layers and films suspended in a viscous fluid. Although the viscosity of the surrounding fluid is usually small, experiments show that the role of the fluid surrounding the layer cannot be neglected, because it acts as an external dissipation mechanism. In turbulent fluxes, it damps the turbulent vortices. Rivera and collaborators [46] phenomenologically modeled this damping by adding a linear drag term in the two-dimensional Navier-Stokes equations. They found the dissipation due to air friction to be a significant energy dissipation mechanism in their experimental system. The dissipation due to air also plays an important role in the mitigation of the divergence determined by the Stokes paradox during diffusion in two-dimensional systems. In the absence of air friction, the mobility of a particle in a 2D system would be infinite. Therefore, a particle subjected to a steady force would accelerate indefinitely: This is the essence the "Stokes paradox" mentioned above, which would lead to an infinite diffusion coefficient. However, the viscous drag exerted by a fluid surrounding the thin film quenches the low-frequency velocity fluctuations and decreases the mobility to a finite value. This was first predicted theoretically by Saffman [53] and later confirmed by experiments on diffusion [52] and Brownian motion [45].

An important biological model system of 2D fluids is represented by lipid bilayers, such as those of the membranes of cells, made by the superposition of two layers of amphiphatic molecules (see, for example, Ref. [36] and references therein). Pioneering experiments provided evidence that lipids and proteins are free to diffuse along the surface of the membrane [54], which acts as a 2D liquid. The membranes are surrounded by a viscous liquid, whose action prevents the Stokes paradox, as first predicted theoretically by Saffman and Delbrock [55]. Cell membranes behave as a multicomponent liquid mixture and they exhibit a complex phase behavior that can be mimicked in the laboratory by using giant unilamellar vescicles or giant plasma membrane vescicles. Experiments show that the vescicles exhibit critical behavior [56,57] in the same universality class of the $2 \mathrm{D}$ Ising model [58]. Below the critical temperature, the phase separation leads to the formation of immiscible lipid domains, which diffuse along the membrane with diffusion coefficients in good agreement with the Saffman-Delbrock model [59]. Above the critical temperature, the lipid domains dissolve. We argue that the diffusive dissolution of the domains could lead to the development of giant nonequilibrium fluctuations. We estimate the amplitude of these fluctuations under realistic conditions found in diffusion experiments $[59,60]$.

Table I summarizes the physical parameters describing the experimental model systems such as liquid crystals and cell membranes surrounded by a less viscous fluid. In the case of layers surrounded by a gaseous phase, when the pressure of the gas is decreased, its viscosity remains almost constant, according to Sutherland law; however, for some liquid crystals that have a low vapor pressure, it is possible to decrease the pressure so much that the "Knudsen" and "molecular" regimes are reached. While the analysis of the Knudsen regime is outside the scope of this paper, in the Appendix we show that, at a suitable pressure and on the length scales relevant for our work, a liquid crystal layer suspended in medium vacuum displays the ideal behavior of a 2D fluid and the friction with the surrounding fluid can be neglected. It is worth noting that, in the "molecular" regime, air cannot be considered a continuous medium and the friction with the gas cannot be described in term of a shear viscosity.

\section{MATHEMATICAL MODEL OF THE NONEQUILIBRIUM FLUCTUATIONS.}

Theoretical work performed since the early 1990s [7] showed that fluctuating hydrodynamics [17] represents a reliable quantitative model to describe nonequilibrium fluctuations generated by small gradients. Fluctuating hydrodynamics relies on a linearization of the Navier-Stokes equations to obtain a set of equations that, once supplemented with stochastic white-noise terms, act as Langevin equations for the relevant thermophysical variables. In this work we are interested mainly in modeling the concentration fluctuations generated by a concentration gradient and we will assume that the system is isothermal, so that the nonequilibrium temperature fluctuations can be neglected. We also neglect the contribution of temperature and concentration equilibrium fluctuations. We do not take into account thermal and solutal capillary effects at the surface of the fluid, because these 
effects do not exist for a truly 2D fluid like monolayers and bilayers [33], due to the absence of a bulk phase. In 2D fluids, the equivalent of the 3D surface tension is the "line tension," which can originate from the presence of separated phases in the liquid mono- or bilayer; in this case, the lines separating the domains tend to minimize the length of the borders. In our case, we consider miscible fluids, in which the gradient of concentration is relatively small; in analogy with $3 \mathrm{D}$ fluctuations [61,62], line tension effects generated by small concentration gradients are negligible.

The velocity correlation function for a two- and threedimensional liquid is [63]

$$
\begin{aligned}
& \left\langle\vec{n} \cdot \vec{u}(\vec{q}, \omega) \vec{n} \cdot \vec{u}^{*}\left(\vec{q}^{\prime}, \omega^{\prime}\right)\right\rangle \\
& \quad=\delta\left(\vec{q}-\vec{q}^{\prime}\right) \delta\left(\omega-\omega^{\prime}\right) \frac{K_{B} T v}{8 \pi^{4} \rho} \frac{q^{2}-(\vec{q} \cdot \vec{n})^{2}}{\omega^{2}+v^{2} q^{4}},
\end{aligned}
$$

where $\vec{u}$ is the hydrodynamic velocity, $n$ is a generic unit vector, and the wave vector $q$ is in $\mathbb{R}^{2}$ or $\mathbb{R}^{3}$ depending on the dimensionality of the system. It should be noticed that in two dimensions $\rho$ is the surface mass density.

The velocity correlation function for a liquid layer, taking into account the viscous drag exerted by the surrounding fluids, is the sum of two functions of $\omega$, with characteristic decay times associated to the viscous damping inside the liquid layer and by the friction with the surrounding fluids [64]. Here we report only the value of the velocity correlation function for $\omega=0$, which will be used in the following:

$$
\begin{aligned}
& \left\langle\vec{n} \cdot \vec{u}(\vec{q}, \omega=0) \vec{n} \cdot \vec{u}^{*}\left(\vec{q}^{\prime}, \omega^{\prime}\right)\right\rangle \\
& \quad=\delta\left(\vec{q}-\vec{q}^{\prime}\right) \delta\left(\omega^{\prime}\right) \frac{K_{B} T}{8 \pi^{4} \rho \nu_{0}} \frac{q^{2}-(\vec{q} \cdot \vec{n})^{2}}{q^{3}\left(q_{C}+q\right)},
\end{aligned}
$$

where the characteristic wave vector $q_{C}$ is defined by:

$$
q_{C}=\frac{2 \eta_{1}}{h \eta_{0}}
$$

where $\eta_{0}$ and $\eta_{1}$ are the shear viscosities of the layer and of the surrounding fluid, respectively, $\rho_{0}$ and $\rho_{1}$ the volumetric densities, $\rho=\rho_{0} h$ is the surface density, and $h$ the thickness of the layer. $q_{C}$ represents the characteristic wave vector, below which the velocity fluctuations are damped by the viscous drag of the surrounding fluids.

The time evolution of the concentration $c(\vec{x}, t)$ is determined by advection and diffusion:

$$
\frac{\partial}{\partial t} c(\vec{x}, t)=-\vec{u}(\vec{x}, t) \cdot \vec{\nabla} c(\vec{x}, t)+D \nabla^{2} c(\vec{x}, t),
$$

where $\vec{u}(\vec{x}, t)$ is the velocity field and $D$ is the diffusion coefficient. The vectors can be in two or three dimensions, depending on the dimensionality of the physical system.

We assume that the concentration gradient is not significantly affected by the fluctuations and is constant in space and time; this implies that the macroscopic concentration gradient is always much more intense than the amplitude of fluctuations. This assumption is quite strong, since, as we will show, in a 2D liquid film or membrane the fluctuations can become of the order of $10 \%$ of the macroscopic concentration differences.

In Fourier space:

$$
-i \omega c(\vec{q}, \omega)=-\vec{u}(\vec{q}, \omega) \cdot \vec{\nabla} c_{0}-q^{2} \operatorname{Dc}(\vec{q}, \omega) .
$$

By solving with respect to the concentration we get:

$$
c(\vec{q}, \omega)=-\frac{\vec{u}(\vec{q}, \omega) \cdot \vec{\nabla} c_{0}}{-i \omega+q^{2} D} .
$$

From this expression, we obtain the correlation function:

$$
\left\langle c(\vec{q}, \omega) c^{*}\left(\overrightarrow{q^{\prime}}, \omega^{\prime}\right)\right\rangle=\nabla c_{0}^{2} \frac{\left\langle\overrightarrow{\hat{z}} \cdot \vec{u}(\vec{q}, \omega) \overrightarrow{\hat{z}} \cdot \vec{u}^{*}\left(\vec{q}^{\prime}, \omega^{\prime}\right)\right\rangle}{D^{2} q^{4}+\omega^{2}},
$$

where $\vec{z}$ is the unit vector pointing in the direction of the concentration gradient, which is assumed to be parallel to the $z$ axis.

The static power spectrum of the fluctuations can be derived by integrating over $\omega$. The calculation is performed assuming that the diffusion time is much longer than the viscous time: This approximation holds true for $D \ll v$, a condition fulfilled by most binary liquid mixtures (further details are discussed in Ref. [6]),

$\left\langle c(\vec{q}, t) c^{*}\left(\overrightarrow{q^{\prime}}, t\right)\right\rangle=\nabla c_{0}^{2} \frac{\pi}{D q^{2}} \int\left\langle\vec{z} \cdot \vec{u}(\vec{q}, \omega=0) \vec{z} \cdot \vec{u}^{*}\left(\vec{q}^{\prime}, \omega^{\prime}\right)\right\rangle d \omega^{\prime}$.

In the case of two- and three-dimensional fluids, we use Eq. (1) and we obtain [6,21]:

$$
\left\langle c(\vec{q}, t) c^{*}\left(\vec{q}^{\prime}, t\right)\right\rangle=\delta\left(\vec{q}-\vec{q}^{\prime}\right) \frac{K_{B} T}{8 \pi^{3} \rho} \nabla c^{2} \frac{1}{v D q^{4}} \frac{q^{2}-(\vec{q} \cdot \overrightarrow{\hat{z}})^{2}}{q^{2}} .
$$

For the case of a liquid layer with viscous drag of the surrounding fluids, we use Eq. (2) for expressing the velocity correlation function needed by Eq. (8):

$$
\begin{aligned}
& \left\langle c(\vec{q}, t) c^{2}\left(\vec{q}^{\prime}, t\right)\right\rangle \\
& =\delta\left(\vec{q}-\vec{q}^{\prime}\right) \frac{K_{B} T}{8 \pi^{3} \rho} \nabla c_{0}^{2} \frac{1}{v_{0} D q^{3}\left(q_{C}+q\right)} \frac{q^{2}-(\vec{q} \cdot \overrightarrow{\hat{z}})^{2}}{q^{2}} .
\end{aligned}
$$

\section{FLUCTUATIONS AS THE ORIGIN OF FICK'S FLUX}

The presence of a macroscopic concentration gradient inside a binary liquid mixture generates a diffusive mass flux called Fick's flux $\vec{\Phi}=-D \nabla c$ [1]. In fluctuating hydrodynamic theory, the Fick's flux $\vec{\Phi}$ can be interpreted as a fluctuation of flux with a nonvanishing average [21]:

$$
\vec{\Phi}=\langle c(\vec{x}=0, t=0) \vec{u}(\vec{x}=0, t=0)\rangle .
$$

The only nonvanishing component of $\vec{\Phi}$ is along the macroscopic concentration gradient $\nabla c$, directed as the unit vector $\overrightarrow{\hat{z}}$. We express the fields in terms of their Fourier transform:

$$
\overrightarrow{\hat{z}} \cdot \vec{\Phi}=\int\left\langle c(\vec{q}, \omega) \vec{z} \cdot \vec{u}^{*}\left(\vec{q}^{\prime}, \omega^{\prime}\right)\right\rangle d \vec{q} d \vec{q}^{\prime} d \omega d \omega^{\prime} .
$$

We express $c(\vec{q}, \omega)$ by using Eq. (6):

$$
\overrightarrow{\hat{z}} \cdot \vec{\Phi}=-\nabla c \int \frac{1}{-i \omega+q^{2} D}\left\langle\overrightarrow{\hat{z}} \cdot \vec{u}(\vec{q}, \omega) \overrightarrow{\hat{z}} \cdot \vec{u}^{*}\left(\vec{q}^{\prime}, \omega^{\prime}\right)\right\rangle d \vec{q} d \vec{q}^{\prime} d \omega d \omega^{\prime} .
$$

We take into account that the imaginary part of the integrand is an odd function and we assume that the correlation time of 
the velocity fluctuations is much shorter than the diffusive time. Under this assumption, the velocity fluctuations can be considered instantaneous and the velocity correlation is almost constant (approximately equal to its value at $\omega=0$ ) for the values of $\omega$ for which the Lorentzian term under the integral does not vanish. We get:

$$
\begin{aligned}
\overrightarrow{\hat{z}} \cdot \vec{\Phi}= & -\nabla c \int \frac{q^{2} D}{\omega^{2}+q^{4} D^{2}}\left\langle\overrightarrow{\hat{z}} \cdot \vec{u}(\vec{q}, \omega=0) \vec{z} \cdot \vec{u}^{*}\left(\vec{q}^{\prime}, \omega^{\prime}\right)\right\rangle \\
& \times d \vec{q} d \overrightarrow{q^{\prime}} d \omega d \omega^{\prime},
\end{aligned}
$$

The integration over $\omega$ yields:

$$
\overrightarrow{\hat{z}} \cdot \vec{\Phi}=-\nabla c \pi \int\left\langle\overrightarrow{\hat{z}} \cdot \vec{u}(\vec{q}, \omega=0) \overrightarrow{\hat{z}} \cdot \vec{u}^{*}\left(\vec{q}^{\prime}, \omega^{\prime}\right)\right\rangle d \vec{q} d \vec{q}^{\prime} d \omega^{\prime} .
$$

By introducing the diffusion coefficient defined by

$$
D=\pi \int_{Q}^{\Theta}\left\langle\overrightarrow{\hat{z}} \cdot \vec{u}(\vec{q}, \omega=0) \overrightarrow{\hat{z}} \cdot \vec{u}^{*}\left(\vec{q}^{\prime}, \omega^{\prime}\right)\right\rangle d \vec{q} d \overrightarrow{q^{\prime}} d \omega^{\prime},
$$

Equation (15) becomes formally identical to Fick's law. Here we introduced the cut-off wave vectors $\Theta$ and $Q$, associated to modulations at the molecular scale and at the macroscopic size of the fluid system, respectively.

By using Eq. (1) for calculating $D$ with Eq. (16):

$$
D=\frac{K_{B} T}{8 \pi^{3} \rho \nu} \int_{Q}^{\Theta} \frac{q^{2}-(\vec{q} \cdot \hat{z})^{2}}{q^{4}} d \vec{q} .
$$

For the three-dimensional case, we get

$$
D_{3 D}=\frac{K_{B} T}{3 \pi^{2} \rho \nu}(\Theta-Q)
$$

and for the two-dimensional case

$$
D_{2 D}=\frac{K_{B} T}{8 \pi^{2} \rho v_{0}} \log \frac{\Theta}{Q} .
$$

By using Eq. (2) for calculating $D$ with Eq. (16) for the case of the liquid layer with viscous drag:

$$
D=\frac{K_{B} T}{8 \pi^{3} \rho v_{0}} \int_{Q}^{\Theta} \frac{q^{2}-(\vec{q} \cdot \hat{z})^{2}}{q^{3}\left(q+q_{C}\right)} d \vec{q} .
$$

By integrating:

$$
D_{V D}=\frac{K_{B} T}{8 \pi^{2} \rho v_{0}} \ln \frac{\Theta+q_{C}}{Q+q_{C}} .
$$

The integrals leading to Eqs. (18), (19), and (21) are graphically represented in Fig. 1. One can appreciate that, in all the cases, the integrals diverge for increasing values of the high-wavelength cutoff $\Theta$. In the three-dimensional case, only the short-wavelength components contribute to diffusion. For example, from Eq. (18) one can see that $90 \%$ of the integral from 0 to $\Theta$ is reached by integrating over the wavelengths from $\Theta / 10$ to $\Theta$, i.e., by considering only the length scales up to 10 times larger than the molecular radius $a$ : in three dimensions, diffusion is a process that mainly involves the molecular length scales. We can thus take the limit for $Q \rightarrow 0$ of Eq. (18). Moreover, by introducing a molecular length scale defined as $a=\pi /(2 \Theta)$, we find the well-known

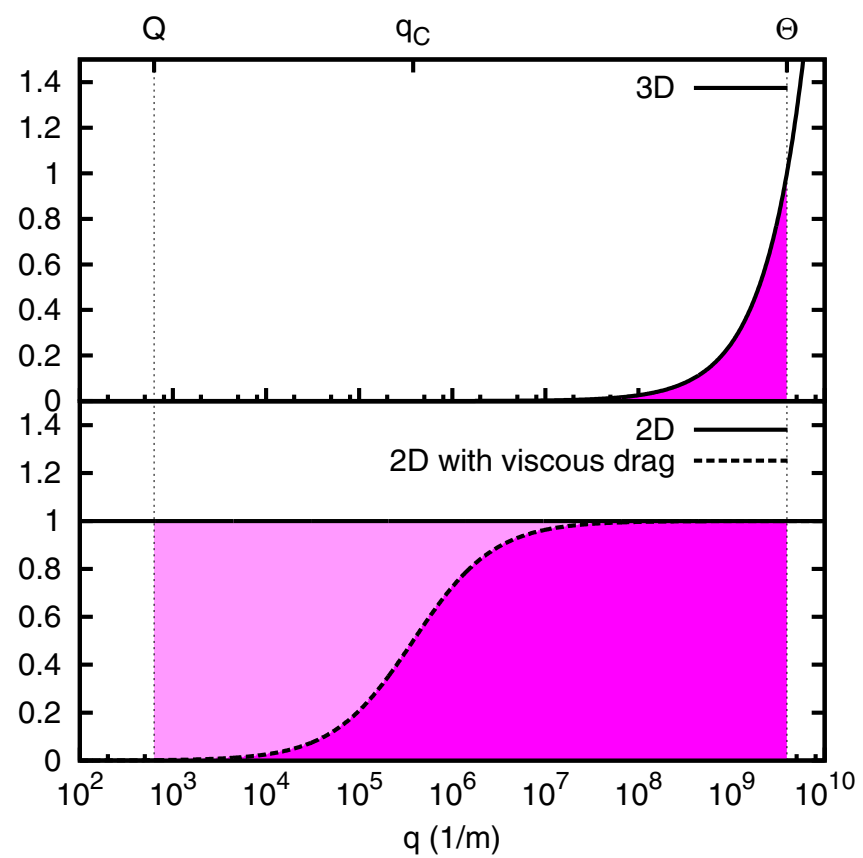

FIG. 1. Graphical representation of the diffusion coefficient as the integral of the velocity power spectrum for $\omega=0$. The top and bottom panels correspond to the $3 \mathrm{D}$ and $2 \mathrm{D}$ cases, respectively. The diffusion coefficient is the area below the curve, between the lowand high-wave-vector cutoffs $Q$ and $\Theta$. The parameters are $Q=$ $2 \pi / 1 \mathrm{~cm}, a=0.4 \mathrm{~nm}, \Theta=\pi /(2 a), q_{C}=3.8 \times 10^{5} \mathrm{~m}^{-1}$.

Stokes-Einstein formula [65]:

$$
D_{3 D}=\frac{K_{B} T}{6 \pi \eta a} .
$$

The numerical constants in this formula depend on the arbitrary choice of the relation between $a$ and $\Theta$. However, the procedure can be made rigorous by means of a renormalization approach [21].

In the two-dimensional case, the integral diverges for $Q \rightarrow$ 0 . This is the consequence of the "Stokes paradox" [53], which gives rise to the divergence of the mobility of a particle in a twodimensional liquid layer when the surface of the layer becomes infinite. In this two-dimensional case, the long wavelengths are not only relevant but even determine a divergence. In practice, the finite size of the system determines a cutoff,

$$
Q=\pi / L,
$$

where $L$ is the macroscopic size of the liquid film, in Eq. (19):

$$
D_{2 D}=\frac{K_{B} T}{4 \pi \rho v_{0}} \log \frac{L}{a} .
$$

This ideal 2D case can be experimentally realized with a liquid crystal monolayer suspended in medium vacuum, at least on the length scales relevant for the present work. When the layer is surrounded by a lower viscosity fluid, the viscous drag it exerts on the film damps the velocity fluctuations at wave vectors shorter than $q_{C}$. In this case, macroscopic length scales up to $2 \pi / q_{C}$ contribute to diffusion, which cannot be considered any more a molecular process. Due to the damping, it is, however, possible to take the limit $Q \rightarrow 0$ of Eq. 21; this 
yields a value of the diffusion coefficient that is of the same order of magnitude of the well-known Saffman formula for the diffusion coefficient of a particle on a liquid film [45,52,53]:

$$
D_{V D}=\frac{K_{B} T}{4 \pi \rho v_{0}} \ln \frac{2}{\gamma a q_{C}},
$$

where $\gamma \approx 0.577$ is the Euler-Mascheroni constant.

\section{ROOT-MEAN-SQUARE AMPLITUDE OF FLUCTUATIONS}

The root-mean-square value of the fluctuations of concentration is calculated by integrating the power spectrum. The procedure is similar to that followed in the three-dimensional case [19]. The calculation requires the introduction of a small-wavelength cutoff $Q$. We assume that the cut-off wave vector is determined by the finite size of the system, as defined in Eq. (23); we consider a system with a constant concentration difference $\Delta c$ at the boundaries, with a concentration gradient $\nabla c=\Delta c / L$, where $L$ is the size of the region over which the concentration gradient is present. For three-dimensional fluids, the root-mean-square amplitude of the fluctuations is [19]

$$
c_{\mathrm{rms}}=\Delta c \sqrt{\frac{K_{B} T}{3 \pi^{3} \rho L \nu D}} .
$$

This expression can be further elaborated by using the StokesEinstein relation [65] (Eq. (22)) to write $D$ in terms of the viscosity $v$ and the radius $a$ of the diffusing particles to obtain:

$$
c_{\mathrm{rms}}=\Delta c \sqrt{\frac{2}{\pi}} \sqrt{\frac{a}{L}} .
$$

We thus conclude that, for three-dimensional systems, the amplitude of the fluctuations decreases as the macroscopic length scale $L$ of the system increases, with respect to the molecular size $a$. For $L$ of the order of $1 \mathrm{~mm}$ and $a$ of the order of $1 \mathrm{~nm}$, the fluctuation amplitude is of the order of 1/1000 of the macroscopic concentration difference. The corrugation of the diffusion front can be roughly evaluated to be $c_{\mathrm{rms}} / \nabla c \propto$ $\sqrt{a L}$ : It is proportional to the geometric average between the macroscopic length scale $L$ and the molecular size $a$, showing that the phenomenon is actually "mesoscopic" in amplitude, i.e., its amplitude is intermediate between the macroscopic and microscopic length scale [19].

Now we extend the calculation of the root-mean-square value of the fluctuations of concentration to the twodimensional case. By integrating Eq. (9):

$$
\begin{aligned}
c_{\mathrm{rms}}^{2} & =\frac{K_{B} T}{8 \pi^{3} \rho} \nabla c^{2} \frac{1}{\nu D} \int_{0}^{2 \pi} d \theta \int_{Q}^{\infty} q d q \frac{\sin ^{2}(\theta)}{q^{4}}, \\
& =\frac{K_{B} T}{16 \pi^{2} \rho} \nabla c^{2} \frac{1}{\nu D} \frac{1}{Q^{2}} .
\end{aligned}
$$

By introducing the finite-size cutoff defined by Eq. (23):

$$
c_{\mathrm{rms}}=\Delta c \sqrt{\frac{K_{B} T}{8 \pi^{4} \rho \nu D}} .
$$

The parameter $L$ does not explicitly appear in this expression for $c_{\text {rms }}$; however, $D$ depends from $L$, as shown in Sec. IV. We use Eq. (24) to express the diffusion coefficient $D$ in Eq. (30):

$$
c_{\mathrm{rms}}=\frac{\Delta c}{\pi \sqrt{\log \frac{L}{a}}} .
$$

We see that, also in this case, the fluctuation amplitude vanishes as the ratio $L / a$ tends to infinity. However, the dependence is through a logarithm and is thus much slower than in the three-dimensional case [Eq. (27)]. For example, taking again as a reference condition a size of the system $L$ of the order of $1 \mathrm{~mm}$ and a molecular scale $a$ of the order of $1 \mathrm{~nm}$, the two-dimensional fluctuation amplitude is of the order of $10 \%$ of the macroscopic concentration difference.

By approximating the corrugation of the fronts of diffusion with $c_{\mathrm{rms}} / \nabla c$, we get:

$$
h_{\mathrm{rms}}=\frac{L}{\pi \sqrt{\log \frac{L}{a}}} .
$$

We see that the corrugation is a relevant fraction of the thickness of the diffusion layer, e.g., of the order of $10 \%$ under the above-mentioned conditions.

Now we use Eq. (10) to calculate the root-mean-square value of the fluctuations of concentration for a liquid layer with viscous drag exerted by the surrounding fluids:

$$
\begin{aligned}
c_{\mathrm{rms}}^{2} & =\frac{K_{B} T}{8 \pi^{3} \rho} \nabla c^{2} \frac{1}{v_{0} D} \int_{0}^{2 \pi} d \theta \int_{Q}^{\infty} q d q \frac{\sin ^{2}(\theta)}{q^{3}\left(q_{C}+q\right)}, \\
& =\frac{K_{B} T}{8 \pi^{2} \rho} \nabla c^{2} \frac{1}{v_{0} D}\left(\frac{1}{q_{C} Q}-\frac{1}{q_{C}^{2}} \log \frac{Q+q_{C}}{Q}\right) .
\end{aligned}
$$

By using Saffman formula Eq. (25) to calculate the diffusion coefficient we get:

$$
c_{\mathrm{rms}}=\Delta c \frac{1}{\sqrt{2 \pi}} \frac{1}{L \sqrt{\ln \frac{2}{\gamma a q_{C}}}} \sqrt{\frac{L}{\pi q_{C}}-\frac{1}{q_{C}^{2}} \log \left(1+\frac{L q_{C}}{\pi}\right)} .
$$

Depending on whether the finite size cutoff is much larger or smaller than the cutoff due to the surrounding fluids, one can identify two limiting regimes:

(1) In the limit $Q \gg q_{C}$ (e.g., limit of vanishing viscosity of the surrounding fluid):

$$
c_{\mathrm{rms}}=\Delta c \frac{1}{2 \sqrt{\pi^{3}}} \frac{1}{\sqrt{\ln \frac{2}{\gamma a q_{C}}}},
$$

which has a value close to that of Eq. (31), where the damping effect of the surrounding fluid is negligible.

(2) In the limit $Q \ll q_{C}$ (the hydrodynamics is dominated by the viscosity of the surrounding fluid):

$$
c_{\mathrm{rms}}=\Delta c \frac{1}{\sqrt{2 \pi}} \frac{1}{\sqrt{\ln \frac{2}{\gamma a q_{C}}}} \sqrt{\frac{1}{L \pi q_{C}}},
$$

which has a $1 / \sqrt{L}$ dependence similar to Eq. (27) for a $3 \mathrm{D}$ fluid. 


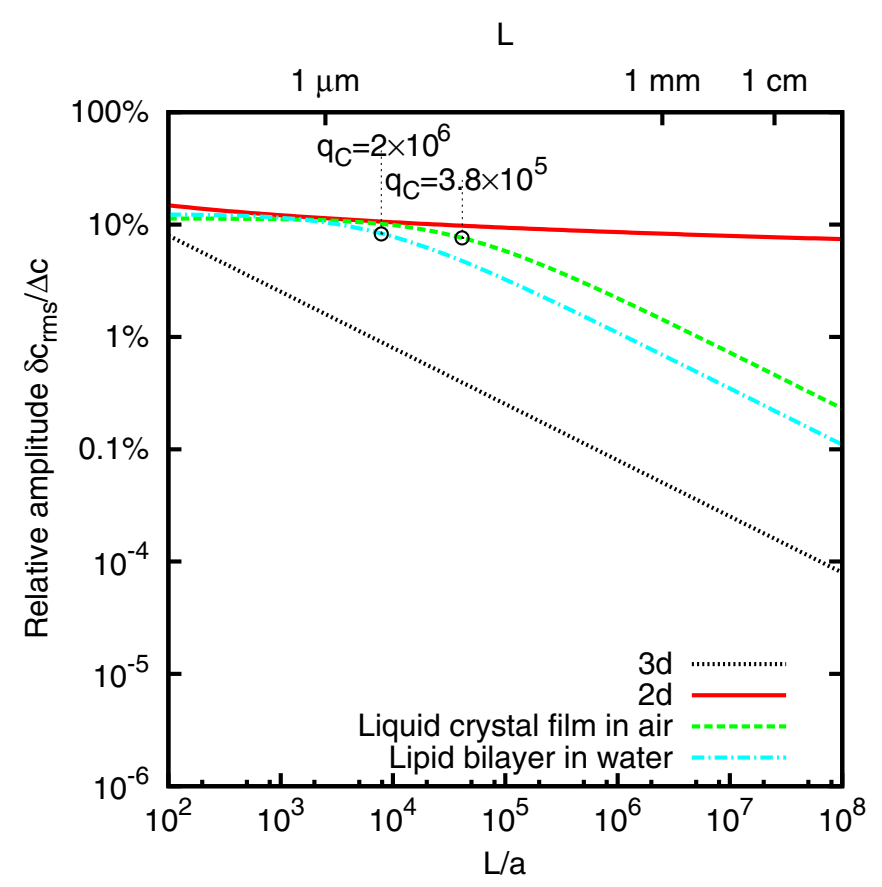

FIG. 2. Root-mean-square amplitude of the nonequilibrium fluctuations of concentration in three-dimensional and two-dimensional fluids and in liquid layers surrounded by less viscous fluids. The amplitude is represented as a fraction of the macroscopic concentration difference $\Delta c$ across the layer of thickness $L$ over which diffusion takes place. The scale on the upper axis assumes $a=0.4 \mathrm{~nm}$. The value of $q_{C}$ is $3.8 \times 10^{5} \mathrm{~m}^{-1}$ and $2 \times 10^{6} \mathrm{~m}^{-1}$, respectively, for the liquid crystal layer suspended in air and for the lipid bilayer suspended in water.

It is interesting to compare the mean-square amplitude of nonequilibrium fluctuations in $2 \mathrm{D}$ and $3 \mathrm{D}$ in the presence of viscous drag by using parameters mirroring those of experimental model systems, such as liquid crystals and cell membranes. Figure 2 shows a comparison between the rootmean-square amplitude of the concentration of nonequilibrium fluctuations in three- and two-dimensional fluids and in liquid layers surrounded by less viscous fluids. The parameters that are used in the calculations are reported in Table I; on the represented length scales, a liquid crystal monolayer suspended in medium vacuum should be a good approximation of a $2 \mathrm{D}$ fluid. It can be clearly appreciated that the fluctuations have a negligible amplitude in three-dimensional fluids, when the macroscopic concentration gradient extends over a thickness of the order of millimeters, such as that usually employed in experiments $[8,9,11,12,16]$. Under the same conditions, the fluctuations are quite intense in the two-dimensional fluids. The viscous drag exerted by the surrounding fluids quenches the fluctuations when the size of the layer becomes larger than the wavelength corresponding to the characteristic wave vector $q_{C}$.

As shown in Table I, in the case of a cell membrane, the critical wave vector is $q_{C} \approx 2 \times 10^{6} \mathrm{~m}^{-1}$. Therefore, when the concentration gradient extends across a region of of space of size smaller than $\lambda_{0} \approx 3 \times 10^{-6} \mathrm{~m}$, nonequilibrium fluctuations in the lipid bilayer become quite large, of the order of $10 \%$ of the concentration difference driving them (Fig. 2). As an example, this condition is met by the lipid domains formed during the phase separation in lipid bilayers. The formation of these domains has been attributed to a second-order phase transition [56,57] giving rise to the phase separation. Once the phase separated bilayer is brought into its one-phase region, the diffusion of the lipid domains should give rise to large amplitude nonequilibrium fluctuations.

Until now, experiments based on the dissolution of the phases generated through a second-order phase transition have been carried out in a critical binary mixture in $3 \mathrm{D}[9,66]$, but so far no evidence of nonequilibrium fluctuations has been reported during the investigation of the dissolution of lipid domains in bilayers mimicking cell membranes. Indeed, the detection of such fluctuations could represent a significant step forward in understanding whether the interactions between the membrane proteins can be affected by Casimir-like forces generated by the nonequilibrium fluctuations. Casimir forces generated by nonequilibrium fluctuations under confinement have been predicted theoretically to exist in three-dimensional fluids [24-26], but never reported experimentally, and no theoretical prediction is currently available for two-dimensional systems.

\section{FRONTS OF DIFFUSION}

The presence of nonequilibrium fluctuations gives rise to corrugated fronts of diffusion, represented by the surfaces where the concentration is constant. In the past, the results of simulations [42,67-69] and experiments [9,12] suggested that the fronts of diffusion could have a fractal structure [43]. In the 3D case, further theoretical analysis showed that the fronts of diffusion do not have a scale-invariant structure [19] but exhibit instead a self-affine structure. In the following, we tackle the problem of the structure of the fronts of diffusion in $2 \mathrm{D}$ by performing simulations providing a detailed graphical representation of the fronts and by showing theoretically that they also have a self-affine structure.

\section{A. Simulation of the fluctuations}

In order to provide a visual comparison between the threeand two-dimensional case, we simulated the nonequilibrium fluctuations; the result is shown in Fig. 3. In the twodimensional cases, the images directly represent the concentration in gray scale and the fluctuation amplitude in false colors; they represent the expected behavior of a liquid crystal layer suspended in medium vacuum. In the three-dimensional case, the images refer to a section of the liquid, parallel to the concentration gradient. To perform simulations we evaluate the power spectrum of the concentration. For the two-dimensional case and for the freely suspended film with viscous drag of the surrounding fluid, Eqs. (9) and (10) directly give the power spectrum of the fluctuations.

For the three-dimensional case, we consider the fluctuation in a section of the liquid with $y=0$. We start from Eq. (9) and we evaluate the concentration correlation along the section:

$$
\begin{aligned}
& \left\langle c\left(q_{x}, y=0, q_{z}, t\right) c^{*}\left(q_{x}^{\prime}, y=0, q_{z}^{\prime}, t\right)\right\rangle \\
& \quad=\int\left\langle c\left(q_{x}, q_{y}, q_{z}, t\right) c^{*}\left(q_{x}^{\prime}, q_{y}^{\prime}, q_{z}^{\prime}, t\right)\right\rangle d q_{y} d q_{y}^{\prime} .
\end{aligned}
$$



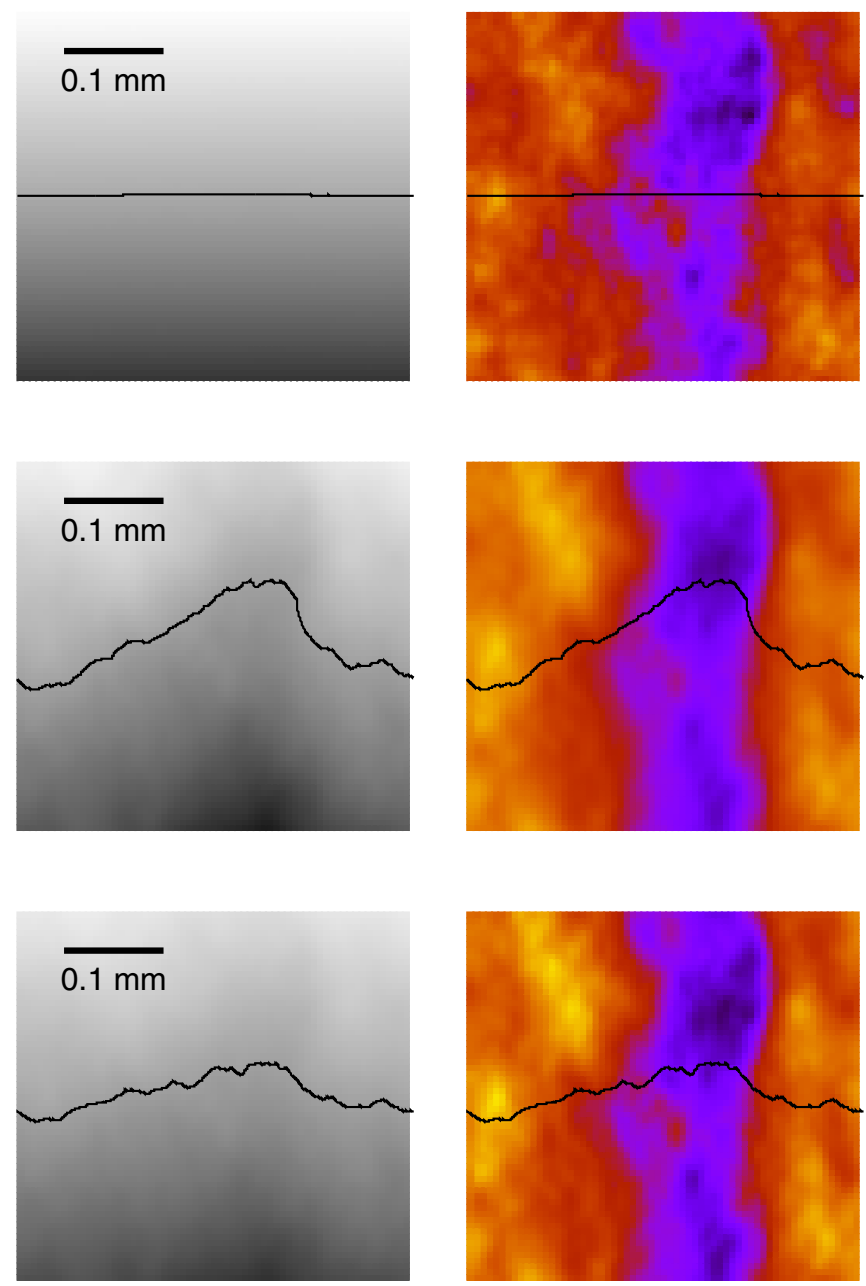

FIG. 3. Sections of the fluid slab where diffusion takes place. For each row, the image on the left is the concentration profile, represented in gray scale; the right image represents the displacement of the concentration with respect to the constant-gradient macroscopic concentration profile. Isoconcentration curves, i.e., the fronts of diffusion, are also shown. The radius of the molecule is $a=0.4 \mathrm{~nm}$ for all the sections. Top row: Three-dimensional fluid. Center row: Two-dimensional fluid; the simulation should be representative of a liquid crystal film suspended in medium vacuum. Bottom row: Liquid layer with viscous drag of the surrounding fluid; the parameters used are those for a lipid bilayer in water.

We get:

$$
\begin{aligned}
& \left\langle c\left(q_{x}, y=0, q_{z}, t\right) c^{*}\left(q_{x}^{\prime}, y=0, q_{z}^{\prime}, t\right)\right\rangle \\
& \quad=\delta\left(q_{x}-q_{x}^{\prime}\right) \delta\left(q_{z}-q_{z}^{\prime}\right) \frac{K_{B} T}{64 \pi^{2} \rho} \nabla c^{2} \frac{1}{v D} \frac{4 q_{x}^{2}+q_{z}^{2}}{\left(q_{x}^{2}+q_{z}^{2}\right)^{5 / 2}} .
\end{aligned}
$$

To create one of the images reported in Fig. 3, we generated a matrix of complex numbers, representing the Fourier transform of the image. For each wave vector, we generate a random number with a Gaussian distribution, with a variance given by the above-reported power spectrum. Applying the inverse Fourier transform then provides the image in real space. The simulation involves all the length scales down to the size of the pixel, i.e., the fluid is considered as continuous across all the length scales that are represented in the picture.

In Fig. 3 one can clearly notice that the fluctuations in the three-dimensional case are barely visible, while they are clearly appreciable in the two other cases. We can also notice that the isoconcentration curves are smoother in the two-dimensional fluid (e.g., liquid crystal film suspended in medium vacuum) than in the suspended film surrounded by a viscous fluid.

\section{B. Hausdorff dimension of the diffusion wave fronts}

Simulations of the fronts of diffusion in three-dimensional fluids suggested that they have a self-similar fractal structure [42,43,67-69]. The power-law behavior exhibited by the static structure factor of concentration fluctuations during diffusion experiments performed on binary liquid mixtures in 3D $[8,9,11,12]$ confirmed that the fronts of diffusion could actually exhibit a scale invariant structure, without, however, providing a quantitative characterization of their fractal dimension. In a recent paper, we have shown theoretically that the fronts of diffusion occurring in three-dimensional systems have instead the self-affine structure of a fractional Brownian surface, with a Hurst exponent $H=1 / 2$ [19].

The Hausdorff dimension of such fractional Brownian surface was found to be 2.5 at small length scales, with a transition to 2 at large length scales [43]. However, this transition takes place around the molecular dimension, and on the range of length scales covered by the fluctuations in real systems the fronts are not fractal $[19,44]$.

In this section, we analyze the case of the two-dimensional fluid. We neglect the viscous drag of the surrounding fluid because it would introduce a characteristic cutoff at $q_{C}$, thus impeding the existence of scaling laws. The discussion is thus relevant for a liquid crystal layer suspended in medium vacuum or below the wave vector $q_{C}$ if the viscous drag is present.

We consider two points inside the solution, displaced along or perpendicularly with respect to the macroscopic concentration gradient, and we evaluate the root-mean-square value of the concentration difference between them. We call the two quantities $\Delta c_{\|}(\Delta z)$ and $\Delta c_{\perp}(\Delta x)$ :

$$
\begin{aligned}
\Delta c_{\|}(\Delta z) & =\sqrt{\left\langle[c(0, t)-c(\overrightarrow{\hat{z}} \Delta z, t)]^{2}\right\rangle}, \\
\Delta c_{\perp}(\Delta x) & =\sqrt{\left\langle[c(0, t)-c(\overrightarrow{\hat{x}} \Delta x, t)]^{2}\right\rangle},
\end{aligned}
$$

where $\overrightarrow{\hat{x}}$ and $\overrightarrow{\hat{z}}$ are the unit vectors perpendicular and parallel to the concentration gradient.

We will see that the integrals leading to such quantities do not diverge: Hence, they are a better local characterization of the fluctuations than the root-mean-square value.

In polar coordinates:

$$
\begin{aligned}
\Delta c_{\|}^{2}(\Delta z)= & \frac{K_{B} T}{8 \pi^{3} \rho} \nabla c^{2} \frac{1}{\nu D} \int_{0}^{2 \pi} d \theta \int_{0}^{\infty} q d q \frac{\sin ^{2}(\theta)}{q^{4}} \\
& \times[2-2 \exp -i q \Delta z \cos \theta],
\end{aligned}
$$




$$
\begin{aligned}
\Delta c_{\perp}^{2}(\Delta x)= & \frac{K_{B} T}{8 \pi^{3} \rho} \nabla c^{2} \frac{1}{v D} \int_{0}^{2 \pi} d \theta \int_{0}^{\infty} q d q \frac{\sin ^{2}(\theta)}{q^{4}} \\
& \times[2-2 \exp -i q \Delta x \sin \theta] .
\end{aligned}
$$

The integrals cannot be easily calculated explicitly. By changing the integration variable to $t=q \Delta x$ and $t=q \Delta z$, we get:

$$
\begin{aligned}
\Delta c_{\|}(\Delta z) & =\sqrt{\frac{K_{B} T}{8 \pi \rho \nu D}} \nabla c \Delta z \Gamma_{\|}, \\
\Delta c_{\perp}(\Delta x) & =\sqrt{\frac{K_{B} T}{8 \pi \rho \nu D}} \nabla c \Delta x \Gamma_{\perp},
\end{aligned}
$$

where

$$
\begin{aligned}
& \Gamma_{\|}=\frac{1}{\pi} \sqrt{\int_{0}^{2 \pi} d \theta \int_{0}^{\infty} d t \frac{\sin ^{2}(\theta)}{t^{3}}[2-2 \exp -i t \cos \theta]} \\
& \Gamma_{\perp}=\frac{1}{\pi} \sqrt{\int_{0}^{2 \pi} d \theta \int_{0}^{\infty} d t \frac{\sin ^{2}(\theta)}{t^{3}}[2-2 \exp -i t \sin \theta]}
\end{aligned}
$$

The integrals converge and can be easily calculated numerically; we obtain the values $\Gamma_{\|} \approx 0.56$ and $\Gamma_{\perp} \approx 0.95$. From Eqs. (43), we can conclude that the fluctuations are a factor 1.7 more elongated along the direction perpendicular to the macroscopic concentration gradient.

Now we evaluate the corrugation of the isoconcentration curves. As we have done above, we approximate the corrugation $h(\Delta x)=c_{\perp}(\Delta x) / \nabla c$. We get:

$$
h(\Delta x)=\sqrt{\frac{K_{B} T}{8 \pi \rho \nu D}} \Gamma_{\perp} \Delta x .
$$

The dependence between $h$ and $\Delta x$ is linear: $h \propto \Delta x$. Therefore, the fronts of diffusion in 2D have the structure of a fractional Brownian process with a Hurst exponent $H=$ 1 [44]. This linear dependence is connected with smoothness, which in this case can be expressed by the Lipschitz condition. With a Hurst exponent equal to 1 , one gets immediately a Hausdorff dimension $d_{f}=2-H=1$, where 2 is the dimensionality of space [44,70-72].

Therefore, similarly to the three-dimensional case, the fronts of diffusion are not fractal, and they exhibit instead a self-affine structure. However, in this case a crossover between a fractal and a nonfractal range of length scales is not present, and the Hurst exponent directly indicates the absence of a scale-invariant structure.

\section{CONCLUSIONS}

Our results allow us to conclude that the amplitude of nonequilibrium fluctuations in two-dimensional fluids is rather large, as it represents a significant fraction of the concentration difference driving diffusion, while they have a negligible amplitude in three-dimensional fluids, when the macroscopic concentration gradient extends over a thickness of the order of millimeters. We suggest that experimental evidence of the existence of concentration nonequilibrium fluctuations in lipid bilayers mimicking the cell membranes could open new venues in the understanding the interaction of macromolecules embedded in membranes. In particular, the theoretical prediction of the existence of nonequilibrium Casimir forces in confined geometries, once confirmed in 2D systems, could represent an important mechanism controlling the interactions in cell membranes.

\section{ACKNOWLEDGMENT}

We thank P. Cicuta and L. Parolini for useful discussion.

\section{APPENDIX : VISCOUS DRAG IN MOLECULAR FLOW REGIME}

In this Appendix we calculate the force exerted by a gas in the molecular flow regime on a moving surface, in analogy with the shear viscosity. We follow a calculation akin to that of the pressure of an ideal gas (made of noninteracting particles); at variance with this case, we have to evaluate the momentum transferred perpendicularly to the surface. We assume completely inelastic collisions. The molecules hitting the surface stick to it, transferring completely their momentum, and are later re-emitted in a different direction, according to Maxwell distribution:

$$
P\left(v_{x}, v_{y}, v_{z}\right)=\left(\frac{m}{2 \pi K_{B} T}\right)^{3 / 2} e^{-\frac{m}{2 K_{B} T}\left(v_{x}^{2}+v_{y}^{2}+v_{z}^{2}\right)},
$$

where $m$ is the mass of a molecule and $v_{x}, v_{y}$, and $v_{z}$ are the componets of its velocity. We consider a surface $S$ on the $x-y$ plane, moving with velocity $v_{0}$ along the $x$ axis. In a time step $\Delta t, N$ particles hit the surface $S$ :

$$
N=\rho S \Delta t \int_{0}^{+\infty} d v_{x} \int_{0}^{+\infty} d v_{y} \int_{0}^{+\infty} d v_{z} v_{x} P\left(v_{x}, v_{y}, v_{z}\right),
$$

where $\rho$ is the number of particles per unit volume. By integrating:

$$
N=\rho S \Delta t \sqrt{\frac{K_{B} T}{2 \pi m}} .
$$

When a molecule hits the surface, it transfers a momentum $m\left(v_{x}-v_{0}\right)$ along the $x$ axis and $m v_{y}$ along the $y$ axis. The average momentum transferred along the $x$ axis is $-m v_{0}$. When the molecule is emitted, the velocity distribution is Maxwellian with respect to the reference system of the moving surface $S, P\left(v_{x}-v_{0}, v_{y}, v_{z}\right)$, so the average transferred momentum is again $-m v_{0}$. The momentum $Q$ transferred by all the hitting molecules is thus

$$
Q=2 m v_{0} \rho S \Delta t \sqrt{\frac{m K_{B} T}{2 \pi}} .
$$

The ratio $Q / \Delta t$ is the force $F$ exerted by the molecules parallel to the surface:

$$
\frac{F}{S v_{0}}=\alpha=2 \rho \sqrt{\frac{m K_{B} T}{2 \pi}} .
$$

The hydrodynamic equation expressing the drag of the gas on the thin film is

$$
\rho_{0} \frac{\partial u}{\partial t}=\eta_{0} h \nabla^{2} u-\nabla p-\alpha u,
$$

where $\rho_{0}$ is the $2 \mathrm{D}$ density of the film, i.e., $\left[\rho_{0}\right]=\mathrm{kg} / \mathrm{m}^{2}$, and $h$ is its thickness. 
The last term of the equation can be neglected for $q>q_{C}$, where

$$
q_{C}=\sqrt{\frac{\alpha}{\eta_{0} h}} .
$$

Air is in the molecular regime on the length scale of $1 \mathrm{~cm}$ at a pressure below $10^{-2} \mathrm{mBar}$. This pressure is larger than the vapor pressure of some liquid crystals [73]. At that pressure $\alpha$ is approximately $1.5 \times 10^{-9} \mathrm{~kg} / \mathrm{m}^{2} \mathrm{~s}$. The resulting $q_{C}$ is of the order of $10 / \mathrm{m}$. Therefore, in the molecular regime, the film is not affected by the presence of the air on the scale of $1 \mathrm{~cm}$ and can be assumed to approach a pure 2D case under experimental conditions.
[1] E. L. Cussler, Diffusion: Mass Transfer in Fluid Systems (Cambridge University Press, Cambridge, 2009).

[2] B. M. Law and J. C. Nieuwoudt, Phys. Rev. A 40, 3880 (1989).

[3] B. M. Law and J. V. Sengers, J. Stat. Phys. 57, 531 (1989).

[4] J. C. Nieuwoudt and B. M. Law, Phys. Rev. A 42, 2003 (1990).

[5] P. N. Segrè and J. V. Sengers, Physica A 198, 46 (1993).

[6] A. Vailati and M. Giglio, Phys. Rev. E 58, 4361 (1998).

[7] J. M. O. de Zárate and J. V. Sengers, Hydrodynamic Fluctuations in Fluids and Fluid Mixtures (Elsevier, Amsterdam, 2006).

[8] A. Vailati and M. Giglio, Phys. Rev. Lett. 77, 1484 (1996).

[9] A. Vailati and M. Giglio, Nature 390, 262 (1997).

[10] D. Brogioli, A. Vailati, and M. Giglio, Phys. Rev. E 61, R1 (2000).

[11] D. Brogioli, A. Vailati, and M. Giglio, J. Phys.: Condens. Matter 12, A39 (2000).

[12] A. Vailati, R. Cerbino, S. Mazzoni, C. J. Takacs, D. S. Cannell, and M. Giglio, Nat. Commun. 2, 290 (2011).

[13] F. Croccolo, C. Giraudet, H. Bataller, R. Cerbino, and A. Vailati, Microgravity Sci. Technol. 28, 467 (2016).

[14] F. Croccolo, D. Brogioli, A. Vailati, M. Giglio, D. S. Cannell, and S. S. Sadhal, Ann. NY Acad. Sci. 1077, 365 (2006).

[15] F. Croccolo, D. Brogioli, A. Vailati, M. Giglio, and D. S. Cannell, Appl. Opt. 45, 2166 (2006)

[16] F. Croccolo, D. Brogioli, A. Vailati, M. Giglio, and D. S. Cannell, Phys. Rev. E 76, 041112 (2007).

[17] L. D. Landau and E. M. Lifshitz, Fluid Mechanics (Pergamon, New York, 1959).

[18] R. Cerbino, Y. Sun, A. Donev, and A. Vailati, Sci. Rep. 5, 14486 (2015).

[19] D. Brogioli, F. Croccolo, and A. Vailati, Phys. Rev. E 94, 022142 (2016).

[20] P. Baaske et al. Eur. Phys. J. E 39, 119 (2016).

[21] D. Brogioli and A. Vailati, Phys. Rev. E 63, 012105 (2000).

[22] A. Donev, J. B. Bell, A. de la Fuente, and A. L. Garcia, Phys. Rev. Lett. 106, 204501 (2011).

[23] A. Donev, T. G. Fai, and E. Vanden-Eijnden, J. Stat. Mech. (2014) P04004.

[24] T. R Kirkpatrick, J. M O. de Zárate, and J. V. Sengers, Phys. Rev. Lett. 115, 035901 (2015).

[25] T. R. Kirkpatrick, J. M. O. de Zárate, and J. V. Sengers, Phys. Rev. E 93, 012148 (2016).

[26] T. R. Kirkpatrick, J. M. O. de Zárate, and J. V. Sengers, Phys. Rev. E 93, 032117 (2016).

[27] M. E. Fisher and P. G. de Gennes, C. R. Acad. Sci. Paris B 287, 207 (1978).

[28] C. Hertlein, L. Helden, A. Gambassi, S. Dietrich, and C. Bechinger, Nature 451, 172 (2008).

[29] A. Gambassi, C. Hertlein, L. Helden, C. Bechinger, and S. Dietrich, Europhys. News 40, 18 (2009).
[30] G. Grinstein, Scale Invariance, Interfaces and Non-equilibrium Systems (Plenum Press, New York, 2006), pp. 261-293.

[31] O. Reynolds, Phil. Trans. R. Soc. Lond. 177, 157 (1886).

[32] G. Grün, K. Mecke, and M. Rauscher, J.Stat. Phys. 122, 1261 (2006).

[33] F. Brochard, P.G. De Gennes, and P. Pfeuty, J. Phys. 37, 1099 (1976).

[34] F. Jähnig, Biophys. J. 71, 1348 (1996).

[35] F. Schneider, Phys. Rev. E 74, 021709 (2006).

[36] M. Edidin, Nat. Rev. Mol. Cell Biol. 4, 414 (2003).

[37] C. Malinverno et al., Nat. Mater. 16, 587 (2017).

[38] A. Creppy, O. Praud, X. Druart, P. L. Kohnke, and F. Plouraboue, Phys. Rev. E 92, 032722 (2015).

[39] A. Creppy, F. Plouraboue, O. Praud, X. Druart, S. Cazin, and H. Yu, P. Degond, J. R. Soc. Interface 13, 20160575 (2016).

[40] A. Sokolov, I.S. Aranson, J. O. Kessler, and R. E. Goldstein, Phys. Rev. Lett. 98, 158102 (2007).

[41] M. V. Dyke, Perturbation Methods in Fluid Mechanics (Academic Press, New York, 1964).

[42] M. Rosso, J. F. Gouyet, and B. Sapoval, Phys. Rev. Lett. 57, 3195 (1986).

[43] A. Lesne and M. Lagëes, Scale Invariance (Springer, Berlin, 2012).

[44] F. Family and T. Vicsek, eds., Dynamics of Fractal Surfaces (World Scientific, Singapore, 1991).

[45] C. Cheung, Y. H. Hwang, X-1. Wu, and H. J. Choi, Phys. Rev. Lett. 76, 2531 (1996).

[46] M. Rivera and X. L. Wu, Phys. Rev. Lett. 85, 976 (2000).

[47] H. Kellay, X-1. Wu, and W. I. Goldburg, Phys. Rev. Lett. 74, 3975 (1995).

[48] M. A. Rutgers, X. 1. Wu, R. Bhagavatula, A. A. Petersen, and W. I. Goldburg, Phys. Fluids 8, 2847 (1996).

[49] Y. Couder, J. Phys. Lett. 45, 353 (1984).

[50] Y. Couder, J. M. Chomaz, and M. Rabaud, Physica D 37, 384 (1989).

[51] X-1. Wu, B. Martin, H. Kellay, and W. I. Goldburg, Phys. Rev. Lett. 75, 236 (1995).

[52] J. Bechhoefer, J. C. Géminard, L. Bocquet, and P. Oswald, Phys. Rev. Lett. 79, 4922 (1997).

[53] P. G. Saffman, J. Fluid Mech. 73, 593 (1976).

[54] L. D. Frye and M. Edidin, J. Cell Sci. 7, 319 (1970).

[55] P. G. Saffman and M. Delbrück, Proc. Natl. Acad. Sci. USA 72, 3111 (1975).

[56] S. L. Veatch, O. Soubias, S. L. Keller, and K. Gawrisch, Proc. Nat. Acad. Sci. USA 104, 17650 (2007).

[57] S. Veatch, P. Cicuta, P.Sengupta, A. H.-S. D. Holowka, and B. Baird, ACS Chem. Biol. 3, 287 (2008).

[58] A. R. Honerkamp-Smith, P. Cicuta, M. D. Collins, S. L. Veatch, M. den Nijs, M. Schick, and S. L. Keller, Biophys. J. 95, 236 (2008). 
[59] S. K. P. Cicuta and S. Veatch, J. Phys. Chem. B 111, 3328 (2007),

[60] Y. Gambin, R. Lopez-Esparza, M. Reffay, E. Sierecki, N. S. Gov, M. Genest, R. S. Hodges, and W. Urbach, Proc. Natl. Acad. Sci. USA 103, 2098 (2006).

[61] P. Cicuta, A. Vailati, and M. Giglio, Phys. Rev. E 62, 4920 (2000).

[62] P. Cicuta, A. Vailati, and M. Giglio, Appl. Opt. 40, 4140 (2001).

[63] R. D. Mountain and J. M. Deutch, J. Chem. Phys. 50, 1103 (1969).

[64] D. Brogioli, arXiv:1103.4760.

[65] C. Tanford, Physical Chemistry of Macromolecules (Wiley, New York, 1961).

[66] F. Giavazzi, A. Fornasieri, A. Vailati, and R. Cerbino, Eur. Phys. J. E 39, 103 (2016).
[67] A. Bunde and J.-F. Gouyet, J. Phys. A: Math. Gen. 18, L285 (1985).

[68] A. Bunde, J.-F. Gouyet, and M. Rosso, I. Phys. A: Math. Gen. 20, 6127 (1987).

[69] V. C. Chappa and E. V. Albano, J. Chem. Phys 121, 328 (2004).

[70] B. B. Mandelbrot, Phys. Scr. 32, 257 (1985).

[71] H. Takayasu, Fractals in the Physical Sciences (Manchester University Press, Manchester, 1990).

[72] K. Falconer, Fractal Geometry: Mathematical Foundations and Applications, 2nd ed. (Wiley, New York, 2003).

[73] P. L. Poole, C. D. Andereck, D. W. Schumacher, R. L. Daskalova, S. Feister, K. M. George, C. Willis, K. U. Akli, and E. A. Chowdhury, Phys. Plasmas 21, 063109 (2014). 